\title{
Effects of Metformin on Bone-derived Mesenchymal Stromal Cell-breast Cancer Cell Line Interactions
}

\author{
Maryana Teufelsbauer \\ Medical University of Vienna \\ Clemens Lang \\ Medical University of Vienna \\ Adelina Plangger \\ Medical University of Vienna \\ barbara Rath \\ Medical University of Vienna \\ Doris Moser \\ Medical University of Vienna \\ Clemens Staud \\ Medical University of Vienna \\ Christine Radke \\ Medical University of Vienna \\ Christoph Neumayer \\ Medical University of Vienna \\ Gerhard Hamilton ( $\sim$ gerhard.hamilton@toc.lbg.ac.at) \\ Medical University of Vienna
}

\section{Research Article}

Keywords: Breast cancer, Metformin, bone-derived mesenchymal stromal cells, migration assay, adipokines

Posted Date: August 5th, 2021

DOl: https://doi.org/10.21203/rs.3.rs-754543/v1

License: (9) This work is licensed under a Creative Commons Attribution 4.0 International License. Read Full License 


\title{
Effects of Metformin on bone-derived mesenchymal stromal cell-breast cancer cell line interactions
}

\author{
Maryana Teufelsbauer ${ }^{1}$, Clemens Lang ${ }^{2}$, Adelina Plangger ${ }^{3}$, Barbara Rath ${ }^{3}$, Doris Moser ${ }^{4}$, \\ Clemens Staud ${ }^{1}$, Christine Radke ${ }^{1}$, Christoph Neumayer ${ }^{3}$, Gerhard Hamilton ${ }^{3, *}$.
}

${ }^{1}$ Department of Plastic and Reconstructive Surgery, Medical University of Vienna, Vienna, Austria

${ }^{2}$ Department of Trauma Surgery, Sozialmedizinisches Zentrum Ost, Donauspital, Vienna, Austria.

${ }^{3}$ Department of Vascular Surgery, Medical University of Vienna, Vienna, Austria

${ }^{4}$ Department of Cranio, Maxillofacial and Oral Surgery, Medical University of Vienna, Vienna, Austria

*Corresponding author Gerhard Hamilton: gerhard.hamilton@meduniwien.ac.at

\begin{abstract}
Metformin is used to treat patients with diabetes mellitus and that was found to lower the incidence of cancer. The present study investigated the effects of metformin on human bonederived mesenchymal stromal cells (BM-MSC) and their breast cancer cell line interactions. BM-MSCs were tested for growth stimulation and migration controlling activity on four breast cancer cell lines employing MTT tests, migration scratch tests and assays of the expression of adipokines in Western Blot arrays. Compared to breast cancer cell lines, metformin significantly inhibited the proliferation of BM-MSC lines. Pretreatment of BMMSCs with metformin showed variable effects on breast cancer cell lines depending on the specific BM-MSC cancer line combination. Metformin significantly impaired the migration of MDA-MB-231 and MDA-MB-436 in response to conditioned media (CM) of drug pretreated BM-MSCs. Metformin-induced alterations of adipokines by BM-MSC CM indicated increased osteogenic signaling and possibly impairment of metastasis. The anticancer activities of metformin seem to be the result of direct and indirect mechanisms. A lower metformininduced protumor activity of BM-MSCs in the bone microenvironment seem to contribute to the anticancer effects of this drug in breast cancer patients.
\end{abstract}

Keywords Breast cancer; Metformin; bone-derived mesenchymal stromal cells; migration assay; adipokines 


\section{Introduction}

Bone metastasis remains one of the most frequent complications of patients suffering from advanced breast cancer ${ }^{1}$. Patients with bone metastases exhibit high morbidity and mortality partially due to excessive osteoclast-mediated bone resorption. Anti-resorptive treatments, such as bisphosphonates or the denosumab monoclonal antibody, are available to mitigate skeletal-related events including pain, risk of fractures, and hypercalcemia ${ }^{2}$. However, at this stage the disease is not curable and 5-year survival rates for these patients are below $25 \%$. In the bone, disseminated breast cancer cells reside in "metastatic niches," peculiar tumor microenvironments (TMEs) that control cancer cell colonization and dormancy as well as progression to overt metastases. Tumor dormancy comprises a prolonged quiescent state of the tumor cells before disease progression becomes clinically apparent ${ }^{3}$. The TME of the niche comprises hematopoietic stem cells and bone marrow mesenchymal stromal cells (BM-MSCs; osteoblasts, fibroblasts and adipocytes) $)^{4}$. BM-MSCs, including the osteoblasts and fibroblasts, play a decisive role during the early stages of breast cancer bone metastasis such as homing, bone marrow colonization, and potential tumor dormancy. Paracrine signaling factors within the TME and extracellular vesicles (EVs), secreted by resident MSCs, support the initiation of dormancy ${ }^{5}$. EVs, including exosomes and microvesicles, derived from MSCs exert similar effects as their parental cells ${ }^{6}$. MCF7 breast cancer cells treated with MSC-derived EVs exhibited reduced migration and proliferation as well as enhanced adhesion, thus supporting cancer cell dormancy.

MSCs are ubiquitous in connective tissues and are defined by their in vitro characteristics and their ability to influence the function of host tissues ${ }^{7}$. MSCs constitute a form of adult stem cells that possess the potential to differentiate into a multitude of cells such as adipocytes, cartilage cells and fibroblasts ${ }^{8}$. MSCs can migrate and home to inflammatory sites. Furthermore, MSCs are known to migrate into tumor-associated stroma and upregulate antiapoptotic and proliferative genes resulting in tumor progression and a poor prognosis ${ }^{9}$.

Epidemiologic studies demonstrated that the biguanide metformin lowers the cancer incidence and mortality among type 2 diabetic patients ${ }^{10,11}$. The majority of in vitro and in vivo studies suggested anticancer properties of metformin at the cellular level but clinical trials revealed mixed outcomes for various tumor types ${ }^{12}$. Metformin affects a wide range of pathways, including glucose utilization, regulation of insulin levels and others and is associated with alterations in metabolites, growth factors, hormones, inflammatory cells and cytokines ${ }^{13}$. We reported recently that metformin showed minor direct inhibitory effects on breast cancer cell lines but exerted a significant impairment of the growth and adipocytic differentiation of adipose-derived stromal cells (ADSCS). Thus, the anticancer activity of metformin in breast cancer cells seems to be mediated partially by an indirect mechanism that lowers the supportive activity of ADSCs for tumor cells ${ }^{14}$. Metastasis to bone is frequently observed in advanced breast cancer and, locally, malignant cells may be affected by BM-MSCs. In the present study, the potential effects of metformin on BM-MSCs and four breast cancer cell lines were tested employing proliferation and migration assays as well as Western blot adipokine arrays. Inhibitory activities of metformin on BM-MSCs may constitute another mechanism retarding metastasis and progress of breast cancer. 


\section{Materials and Methods}

All materials and methods were acquired respectively performed as described previously ${ }^{14}$. All methods were performed in accordance with the relevant guidelines and regulations.

\subsection{Isolation and characterization of BM-MSCs}

BM-MSCs were recovered from patients undergoing hip replacement with informed consent of the patients according to the Ethics Committee Approval 366/2003 of the Ethics Committee of the Medical University of Vienna, Vienna, Austria. All documents and procedures were done in agreement with this Ethics approval and all samples were anonymized. The study protocol was approved by the Ethics Committee of the Medical University of Vienna, Vienna, Austria. Box chisels were fragmented and the resulting suspension incubated in RPMI-1640 medium (Seromed, Berlin, Germany) supplemented with 30\% fetal bovine serum (Seromed) and antibiotics (Sigma-Aldrich, St. Louis, MO, USA). BM-MSCs that had attached to the tissue culture flasks were further cultivated and expanded ${ }^{14}$. The BM-MSCs were checked by flow cytometry for their expression of CD73, CD90, and CD105 and negative reactivity for CD34 using a Cytoflex Flow Cytometer (Beckman Coulter Germany GmbH, Krefeld, Germany). Antibodies and isotype controls were from Biolegend (San Diego, CA, USA) and secondary reagents from Sigma-Aldrich ${ }^{14}$. Data analysis and histogram overlays were done employing the Kaluza flow analysis software (Beckman Coulter). Conditioned media (CM) of the BM-MSCs were prepared by harvesting supernatants of the cells. ADSCs were prepared as described. Metformin was obtained from Sigma-Aldrich.

\subsection{Breast cancer cell lines}

The breast cancer cell lines MDA-MB-231, MDA-MB-436, T47D and HCC1937 were obtained from the ATCC (Rockville, MD, USA), cultured in RPMI-1640 medium and upon confluence, cells were detached with trypsin/EDTA (Sigma-Aldrich) and cells counted with a LUNA cell counter (Biozym, Vienna, Austria) ${ }^{14}$. The commercial ATCC cell lines MDA-MB-231, MDA-MB436 and T47D have been established from pleural effusions and and HCC1937 from a local tumor.

\subsection{Adipokine Western blot arrays}

A panel of adipokines was analyzed using the ARY024 Proteome Profiler Array (R\&D Systems, Minneapolis, MN, USA) according to manufacturer's instructions. Experiments were done in duplicate. Arrays were evaluated using ImageJ and Origin 9.1 software (OriginLab, Northampton, MA, USA) ${ }^{14}$.

\subsection{Cell proliferation assays}

$1 \times 10^{4}$ cells in $100 \mu \mathrm{l}$ medium were distributed to wells of 96-well microtiter plates (TPP, Trasadingen Switzerland) and ten twofold dilutions of the test compound or conditioned media were added in triplicate as described previously ${ }^{14}$. Assays were at least performed in triplicate. The plates were incubated for four days under tissue culture conditions and viable cells detected using a modified MTT assay (EZ4U, Biomedica, Vienna, Austria). Test results were calculated from dose-response curves using Origin 9.1 software (OriginLab, Northampton, MA, USA). 


\subsection{Scratch migration assay}

Breast cancer cell lines were kept in 6-well plates (TPP) in $3 \mathrm{ml}$ medium until confluency was reached. Then, 2 perpendicular scratches were set to remove cells using a plastic tip and wells were supplemented with $1 \mathrm{ml}$ of control medium or respective BM-MSC-CM and further incubated under tissue culture conditions $^{14}$. Light microscopic pictures were taken (magnification 40x) for 3 successive days and scratch areas not covered by cells calculated by ImageJ software (imagej.net) for several positions.

\subsection{Statistical analysis}

Statistical significance was tested by $t$-tests and $p<0.05$ regarded as significant difference.

\section{Results}

\subsection{Isolation and characterization BM-MSCs}

BM-MSCs were isolated from box chisels of female patients undergoing hip replacement and the phenotypes of the cultivated BM-MSCs were checked by flow cytometry. The BM-MSCs were demonstrated to express CD73, CD90 and CD105 with notable absence of the hematopoietic marker CD34 (fig. 1). This phenotype characterizes mesenchymal stromal cells derived from different tissues.

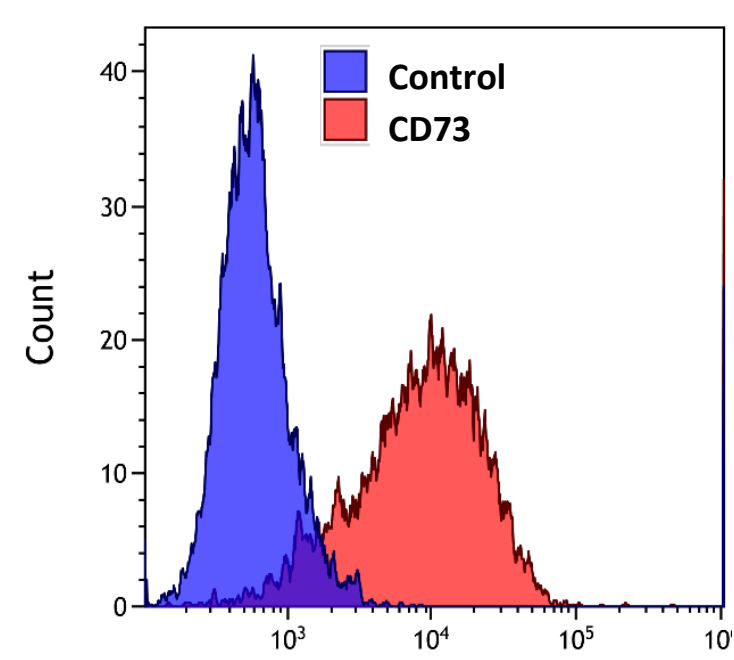

FL1

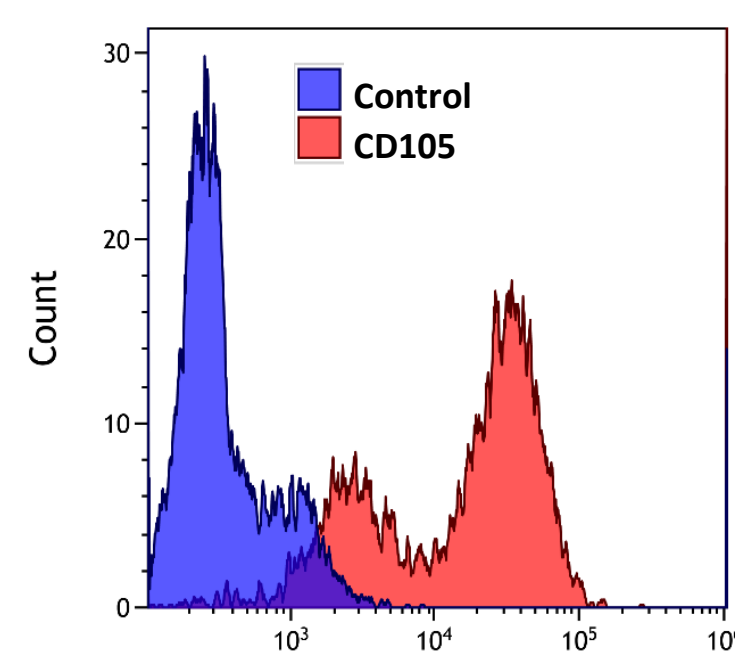

FL1

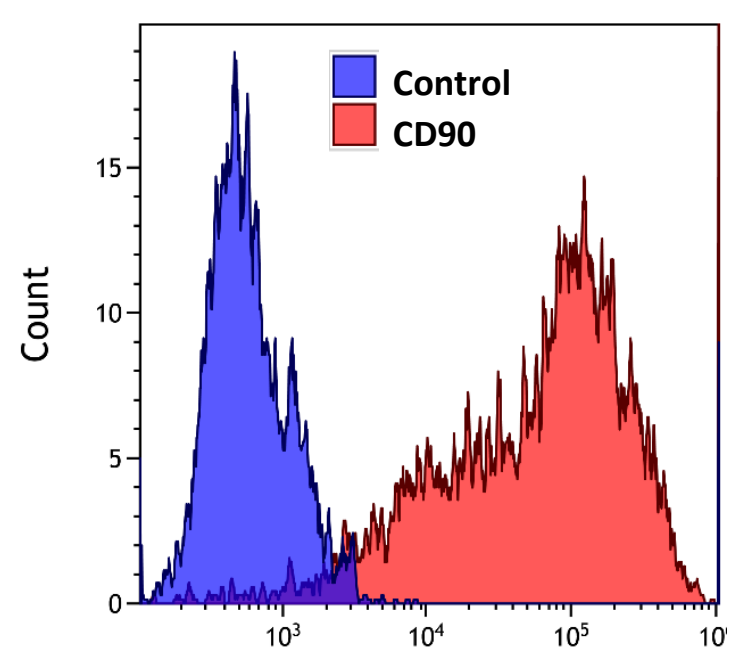

FL1

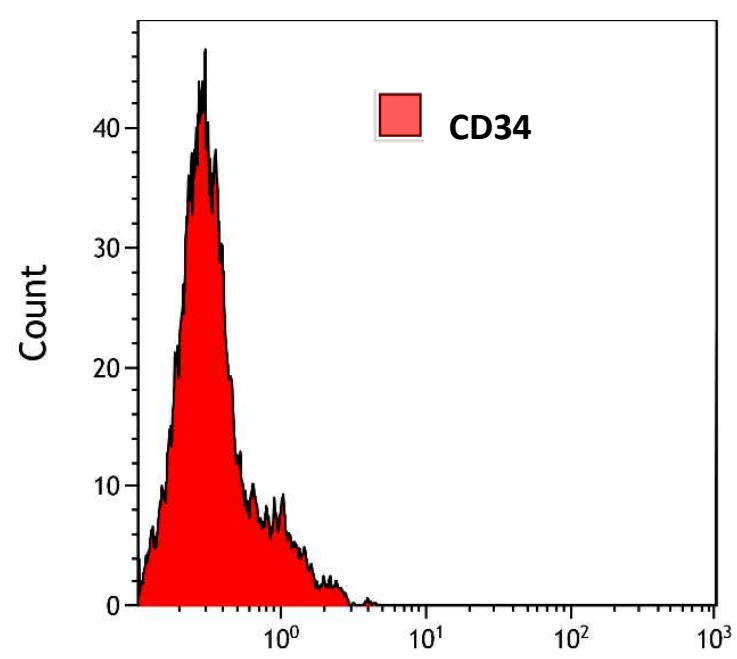

FL1 
Fig. 1 Phenotypic characterization of HIP2 BM-MSC in flow cytometry of cells stained by indirect immunofluorescence for the characteristic MSC markers.

\subsection{Inhibitory effects of metformin on breast cancer cell lines and BM-MSCs}

The growth modulating effects of metformin on 4 breast cancer cell lines, 3 BM-MSCs and one ADSC line were determined employing MTT assays. In controls, metformin showed minor growth-inhibiting effects on the 4 breast cancer cell lines ( $3.6 \pm 1.4 \%$ inhibition) but higher inhibition on the proliferation of HIP2, KM76 and W59 BM-MSCs (12.3 $\pm 2.2 \%$ inhibition). In detail, the inhibitory effect of metformin was $10.5 \pm 3.0$ for HIP2, 5.4 \pm 2.1 for KM76 and 11.0 \pm 2.9 . The ADSC line TRI revealed an inhibitory effect of metformin of $7.9 \pm 3.1 \%$.

\subsection{Effects of metformin on growth-stimulating effects of BM-MSC CM}

Media conditioned by BM-MSCs of different patients stimulated the proliferation of breast cancer cell lines dose-dependently. For example, the responses of two breast cancer cell lines, namely MDA-MB-231 and HCC1937, are shown after exposure to conditioned media of BMMSCs W1951 either as controls or pretreated with $500 \mu \mathrm{M}$ metformin respectively (fig. 2). The CM of the W1951 BM-MSCs exhibits high growth stimulatory effects on both cell lines that were significantly reduced upon preincubation with metformin for 2 days.

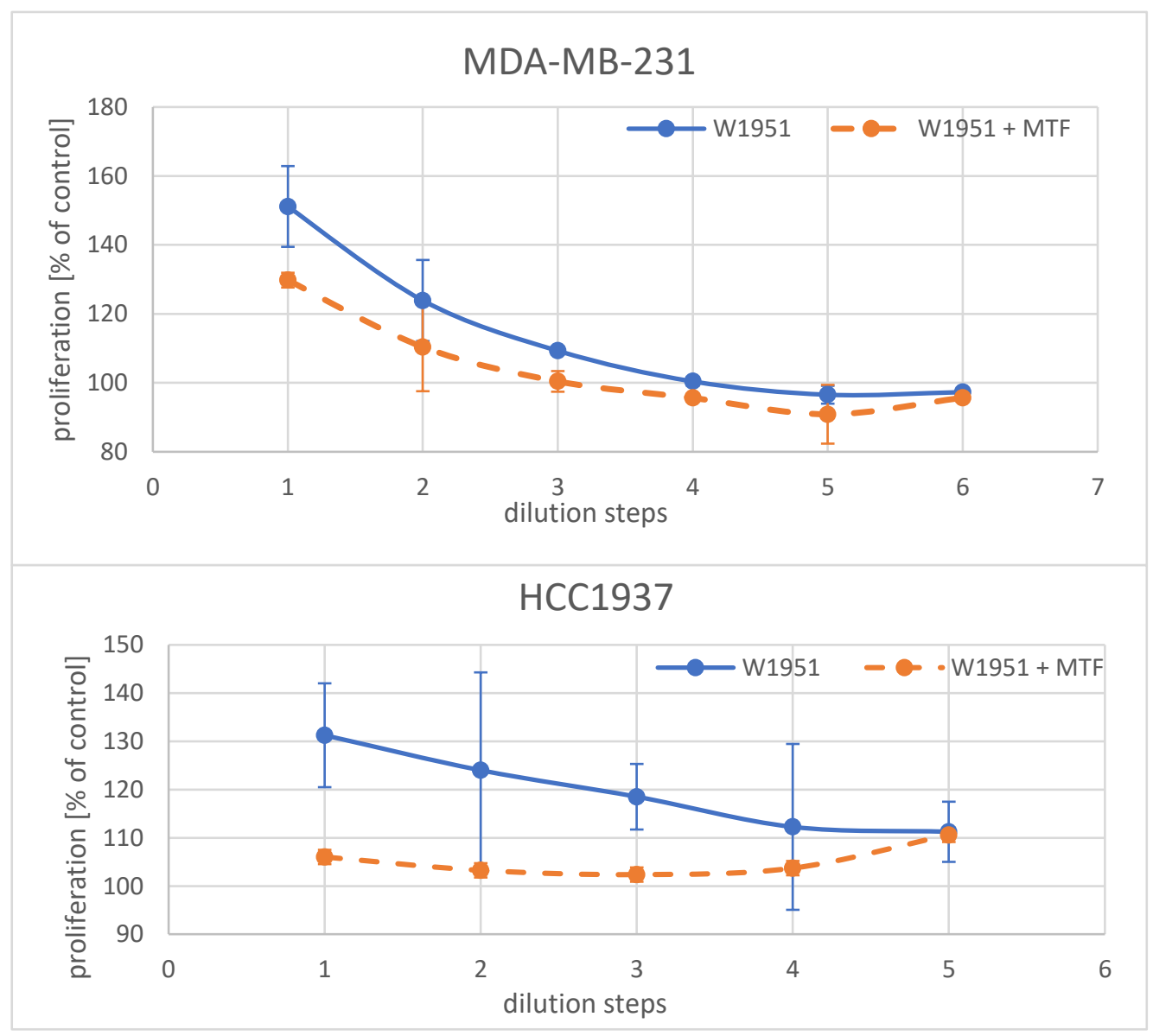


Fig. 2 Effects of CM of W1951 BM-MSCs on cellular proliferation of MDA-MB-231 and HCC1937 breast cancer cells, respectively. Data represent mean values \pm SD. W1951 indicates the control CM and W1951 + MTF the CM of W1051 BM-MSCs pretreated with metformin.

An overview of the effects of control and metformin-pretreated CM of the BM-MSCs and one ADSC line on MDA-MB-231, MDA-MB-436, HCC1937 and T47D breast cancer cell lines is shown in figures $3 \mathrm{~A}$ and $3 \mathrm{~B}$. In general, the exposure of the cells to $500 \mu \mathrm{M}$ metformin resulted in reduced stimulatory activities of the $B M-M S C$ CM dependent on the respective cancer line and BM-MSC combination. For MDA-MB-231, KM76 CM-metformin, W1951 CM-metformin and TRI CM-metformin, the cell proliferation was significantly different from control CM, whereas MDA-MB-436 showed a trend versus inhibitory effects of metformin pretreatment that did not reach statistically significance (3A). In contrast to the other CM-metformin, TRI ADCS CM exhibited stimulation.

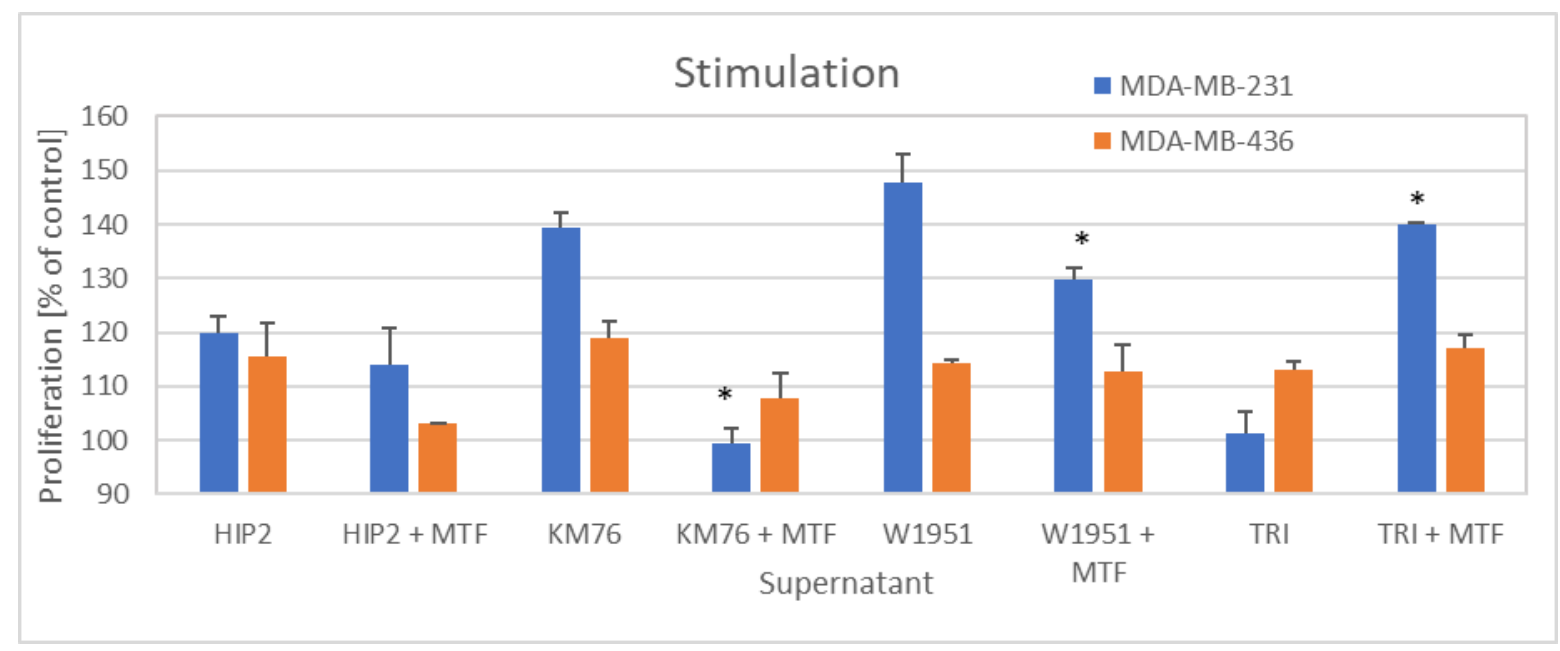

Fig. 3A Cell proliferation assays for MDA-MB-231 and MDA-MB-436 breast cancer cell lines involving control BM-MSC CM and metformin-pretreated BM-MSCs CM, respectively. Data represent mean values \pm SD. Significant differences between control CM and metforminpretreated $\mathrm{CM}$ for the respective cell line are indicated by an asterisk. 


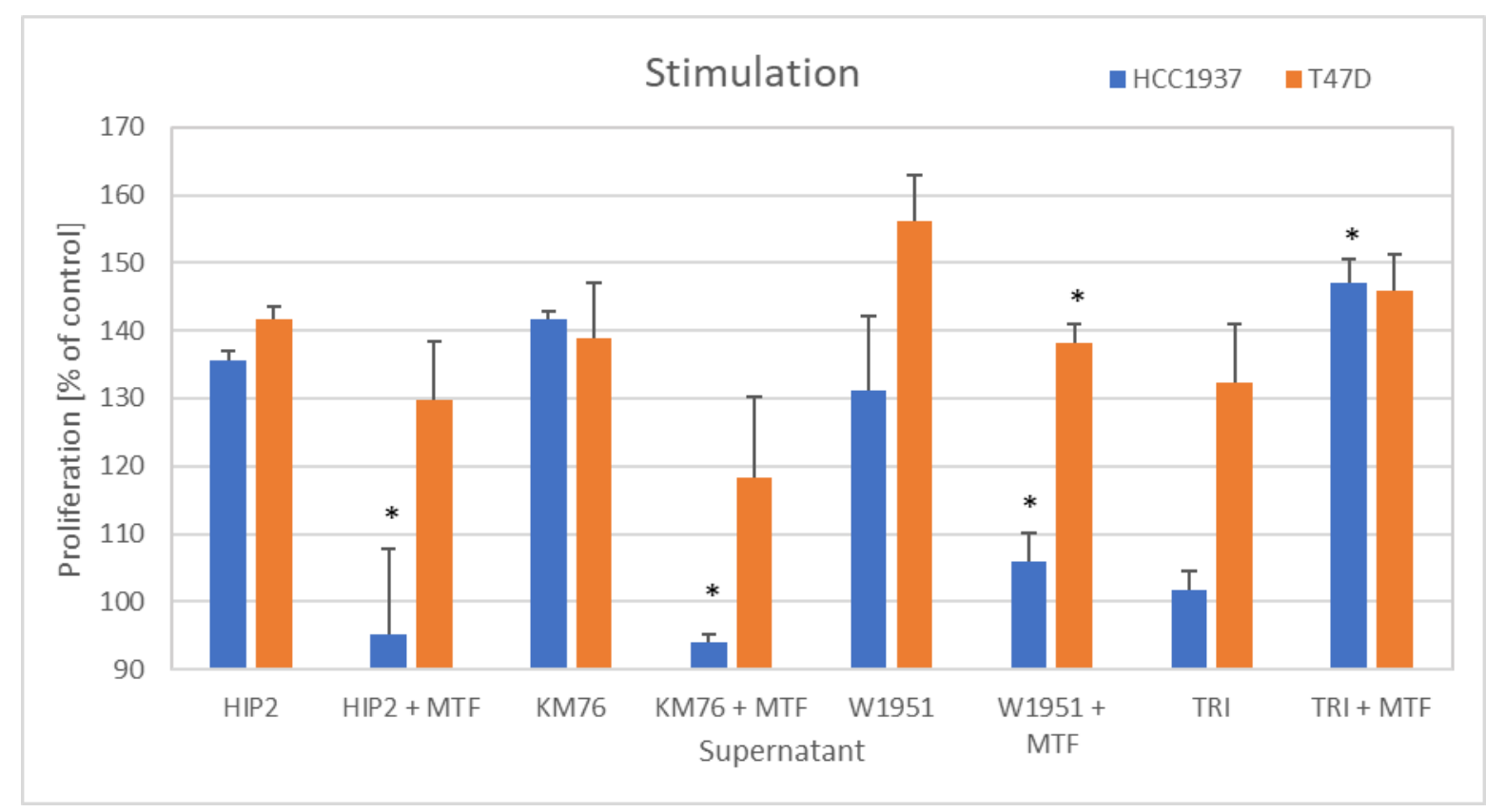

Fig. 3B Cell proliferation assays for HCC1937 and T47D breast cancer cell lines involving control BM-MSC CM and metformin-pretreated BM-MSCs CM. Data represent mean values \pm SD. Significant differences between control $\mathrm{CM}$ and metformin-pretreated $\mathrm{CM}$ for the respective cell line are indicated by an asterisk.

For HCC1937 all differences between BM-MSCs control CM and CM-metformin are significantly different, as well as for W1951 CM and T47D (fig. 3B). Thus, the growth inhibitory effects of metformin are dependent on the specific breast cancer line-BM-MSC combination.

\subsection{Effects of metformin on BM-MSCs CM on migration of the breast cancer cell lines}

Breast cancer cell lines were cultivated in 6-well plates and areas of the confluent cultures removed using a pipette tip. Migration of the cells into the scratch areas were monitored for 2 days and the microscopic pictures evaluated by image analysis. An example depicting a control migration and a CM-supplemented migration assay for MDA-MB-436 breast cancer cells and $\mathrm{KM}-76 \mathrm{BM}-\mathrm{MSC}$ is presented in fig. 4. KM-76 CM reduces the migration of MDA-MB436 to a minor extent but pretreatment of the BM-MSC with metformin resulted in a major inhibition of the migration.
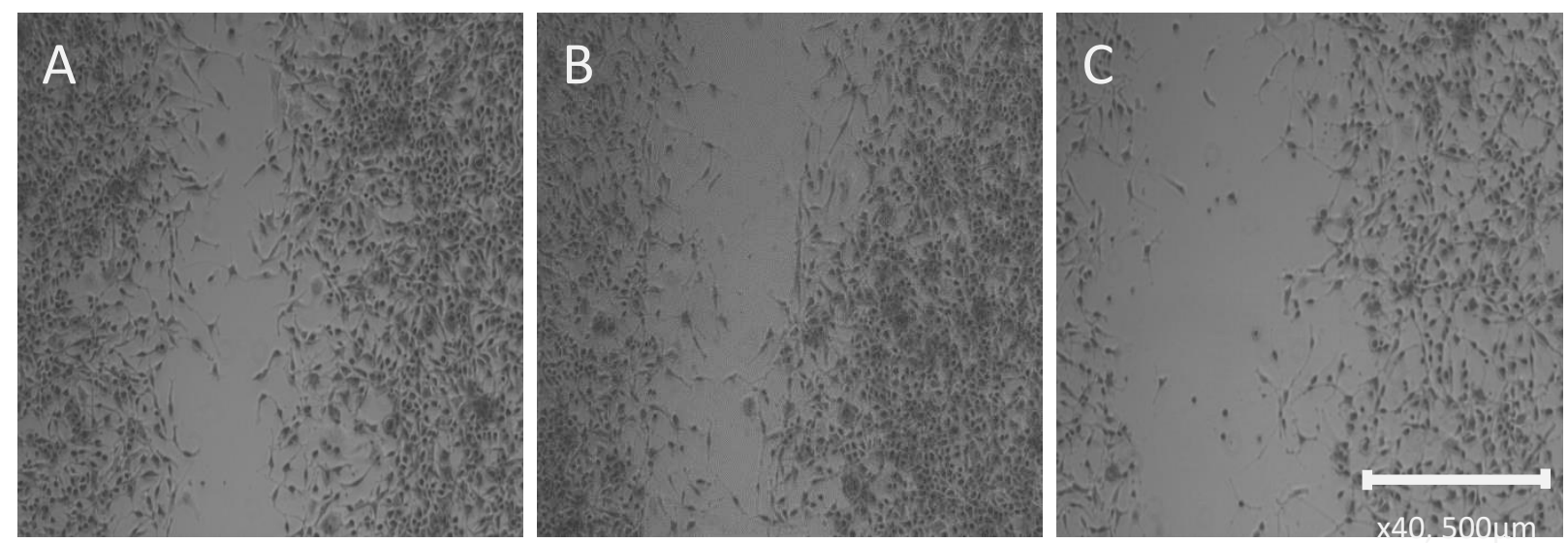
Fig. 4. Migration assay with MDA-MB-436 cells showing the control migration at day $1(A)$, the test in presence of KM76 conditioned medium (B) and in presence of KM76 conditioned medium pretreated with $500 \mu \mathrm{M}$ metformin (C).

Both MDA-MB-231 and MDA-MB-436 breast cancer cell lines show high migration capacity as indicated by a decrease of the blank scratch area on successive days (fig. 5A and B). Pretreatment of the BM-MSCs with metformin and supplementation of the corresponding $\mathrm{CM}$ to scratch assays resulted in a uniform decrease of the migration of MDA-MB-231 and MDA-MB-436 cell lines.

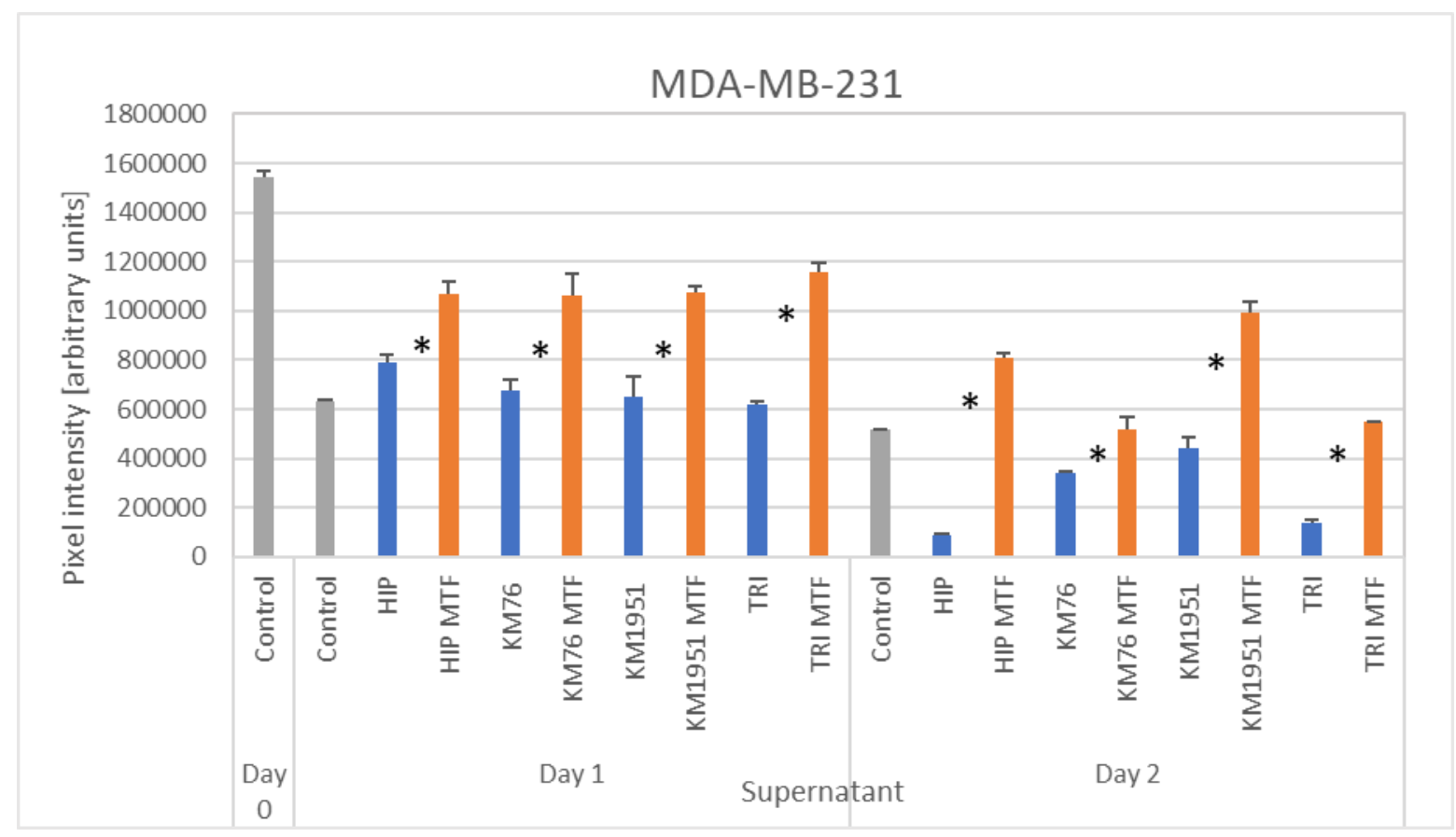

Fig. 5A Migration assay employing MDA-MB-231 cells and control CM and metforminpretreated CM of BM-MSCs and the TRI ADSC. Areas not covered by cells is presented in form of measured pixel intensities and significant differences in the migration of the breast cancer cells in presence of control CM and metformin-pretreated CM for the different BM-MSCs and the ADSC line are indicated by an asterisk between the respective columns. Data presented are mean values \pm SD. 


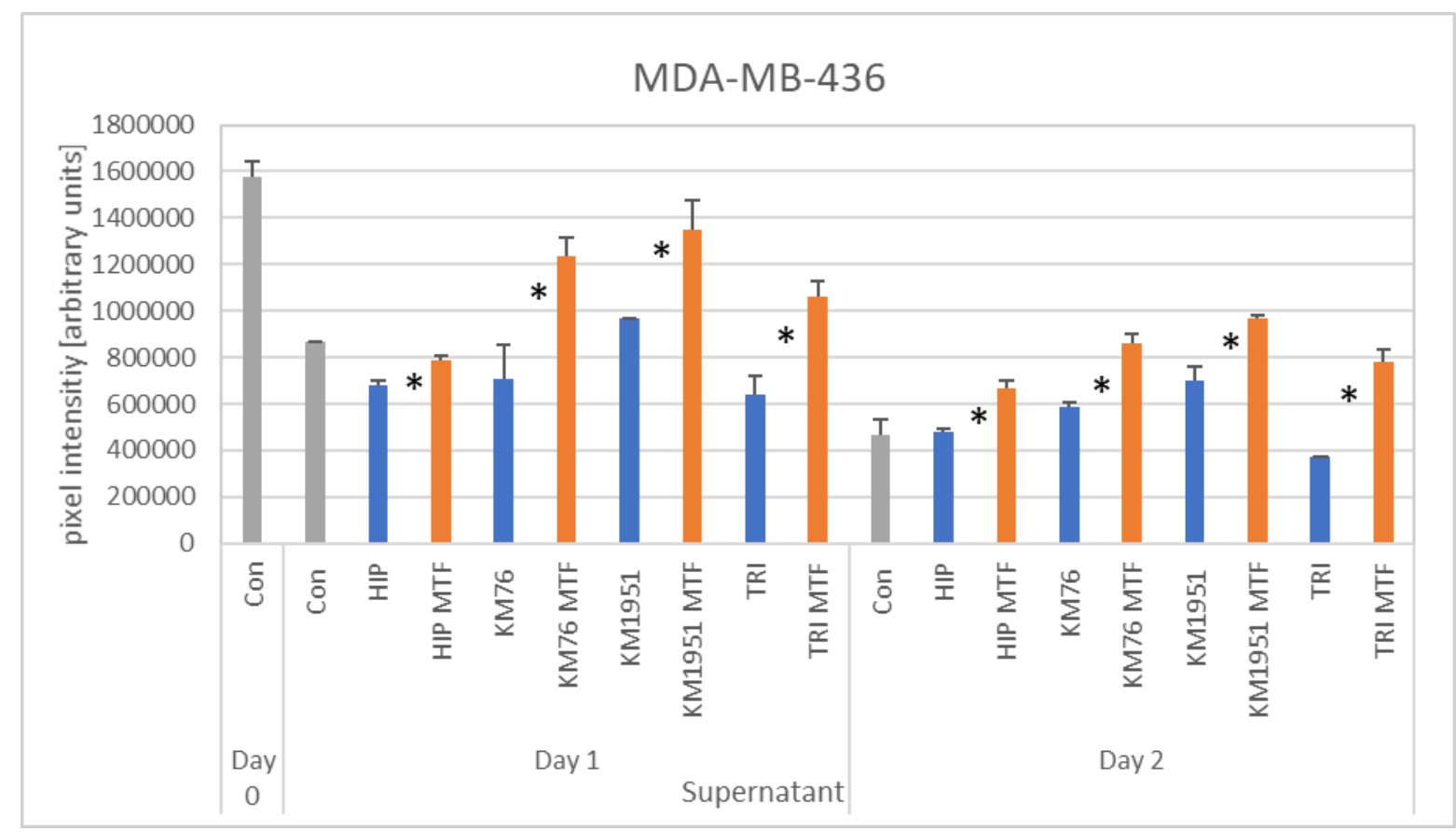

Fig. 5B Migration assay employing MDA-MB-436 cells and control CM and metforminpretreated CM of BM-MSCs and the TRI ADSC. Areas not covered by cells is presented in form of pixel intensities and significant differences in the migration of the breast cancer cells in presence of control CM and metformin-pretreated CM for the different BM-MSCs and the ADSC line are indicated by an asterisk between the respective columns. Data presented are mean values \pm SD.

\subsection{Western blot results of adipokine expression of the BM-MSCs}

Adipokines in CM of BM-MSCs HIP2, KM76, W1951 and ADSC TRI that were pretreated with metformin were determined using Western blot arrays and compared to control CM (fig. $6 \mathrm{~A}$ and $\mathrm{B}$ ). The metformin/control CM ratios show similar characteristics for HIP2 and KM76, whereas W1951 exhibit a divergent adipokine expression pattern in response to pretreatment with metformin. This drug increased the adipokine expression of adiponectin, angiopoietin-2, adipsin and fibrinogen, CD54, RAGE and Rantes in case of HIP2. In case of KM76, the expression pattern is similar to HIP2, except for adipsin, IGFBP-2, visfatin, and TIMP-3. W1951 BM-MSCs overexpress BMP-4, fibrinogen, IL-11, visfatin and TIMP-3 in response to metformin, whereas the expression of the remaining adipokines was reduced with exception of adiponectin and angiopoietin-2 that remained unchanged. ADSCS TRI show downregulation of adiponectin, chemerin, fibrinogen, visfatin and VEGF. In contrast, RANTES and TIMP-3 is overexpressed in response to metformin in TRI. None of the BM-MSCs showed expression of leptin (data not shown). 
Fig. 6A and B Adipokine expression of CM of BM-MSCs and a ADSC line controls and metformin-pretreated cultures were determined with help of Western blot arrays. Data represent mean ratios of the expression of metformin-treated/control $\mathrm{CM} \pm \mathrm{SEM}$ s of selected adipokines.

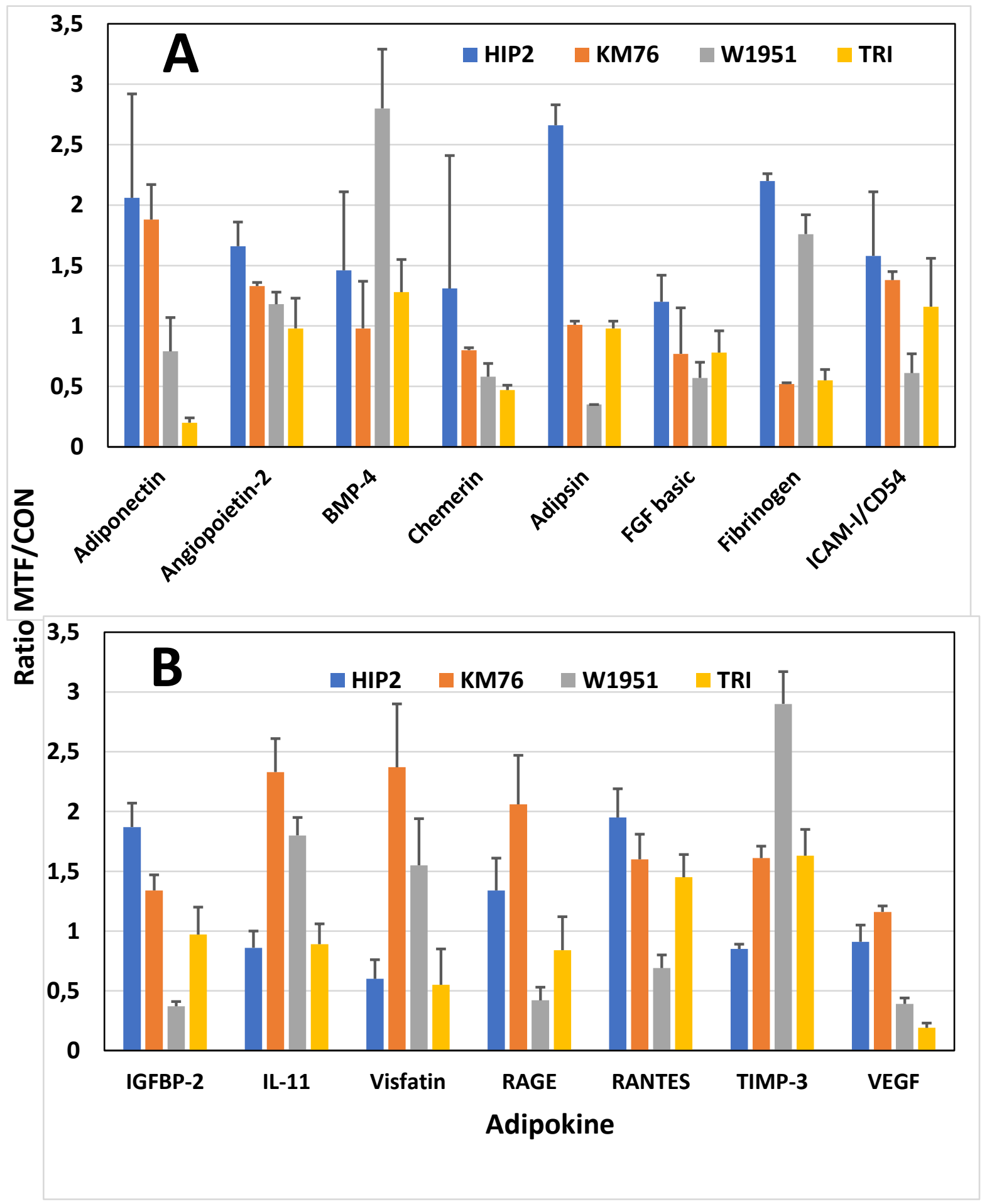




\section{Discussion}

Diabetic patients receiving metformin have been reported to have a lower incidence of breast cancer although a number of studies show divergent results ${ }^{15}$. Metformin affects a host of cellular and physiological mechanisms, including glucose utilization, intracellular AMPK signaling, epithelial-mesenchymal (EMT) signaling, oxidative stress and inflammatory signaling, as well as other pathways 16 . The therapeutic oral dosage of metformin ranges from 500 up to $3000 \mathrm{mg} /$ day depending on the specific disease and maximal metformin levels in human plasma do not exceed $39-50 \mu \mathrm{M})^{17,18}$. There are a host of reports describing anticancer effects of metformin but in many cases concentrations of the drug were used that have no clinical relevance. We have reported recently that ADSCs are significantly more sensitive to metformin than a panel of breast cancer cell lines and that ADSCs seem to impair tumor growth via reduced stimulation by secreted factors ${ }^{14}$. Since bone metastasis is a frequent complication of breast cancer, we investigated the possible effects of metformin on BM-MSCs in the present work.

BM-MSCs were grown from box chisels that were not used during hip replacements for patients with fractures. Disintegrated fragments were kept in tissue culture until outgrowth of cells were observed ${ }^{19}$. Different methods to isolate of BM-MSCs have been published, but simple plating is advantageous compared to other techniques ${ }^{20}$. After expansion, cells were successfully checked for the presence of the typical MSC markers CD73, CD90 and CD105. Donor-matched ADSCs and BM-MSCs populations exhibited characteristics of MSCs, with expression of the typical MSCs markers ${ }^{19}$. In general, ADSCs showed significantly higher proliferation and adipogenic capacity but BM-MSCs possess higher osteogenic and chondrogenic potential.

The first passages of the BM-MSCs $(<5)$ were used to produce CM. CM of the three BMMCSs, namely HIP2, KM76 and W1951, stimulated proliferation of all four breast cancer cell lines significantly (range approximately $120-130 \%$ of medium controls). Pretreatment of the BM-MSC lines with $500 \mu \mathrm{M}$ metformin for 2 days reduced the growth-stimulatory effects of the respective CM in most cases, with highest effects for HCC1937 and MDA-MB-231. For MDA-MB-436 and T47D a similar trend was detected that did reach significance for few selected BM-MSC CM. Thus, the inhibition of the proliferation of breast cancer lines by pretreatment of the BM-MSCs with metformin seems dependent on the specific characteristics of the combination. Furthermore, the effects of the CM of the BM-MSC lines on cell migration was studied for the highly mobile MDA-MB-231 and MDA-MB-436 cell lines, respectively. Metformin-pretreated $\mathrm{CM}$ of all three $\mathrm{BM}-\mathrm{MSC}$ lines inhibited the migration of both MDA-MB-231 and MDA-MB-436 significantly indicating a high indirect impact of metformin on breast cancer mobility. MSCs have been widely implicated in tumor development and metastases and co-culture of BM-MSCs and MDA-MB-231 cells have been found to dramatically reduce the invasiveness of both cell lines when embedded into a matrix ${ }^{21}$.

In breast cancer, adipocytes play an important role in cancer progression, metastasis, and response to treatment. Factors including adiponectin, leptin and others remodel the TME to 
supports the cancer growth ${ }^{22}$. Here, adiponectin is overexpressed in response to metformin in HIP2 and KM76 MSCs. BM-MSCs show expression of the adiponectin receptor and adiponectin stimulates bone formation via induction of osteogenesis-related ${ }^{23,24}$. Mesenchymal progenitors can differentiate into four adipocyte subtypes, one type exhibiting high expression of adiponectin or leptin ${ }^{25}$. In NSCLC cells, exposure to adiponectin increased epithelial markers and decreased mesenchymal markers suggesting lower dissemination ${ }^{26}$. The bone marrow niche cells produce angiopoietin-2 (ANGPT2), that destabilized the local endothelium by impairing ANGPT1/Tie2 signaling and promotes tumor cell survival ${ }^{27}$. BMMSCs are capable of differentiation into osteoblasts, chondrocytes and adipocytes and may undergo spontaneous osteogenic differentiation ${ }^{28}$. Accordingly, BM-MSCs express bone morphogenetic proteins (BMPs) BMP-2, BMP-4 and BMP-6 in addition to cognate receptors and upregulate osteogenic and chondrogenic genes ${ }^{29}$. In particular, BMP-4 enhances epithelial mesenchymal transition (EMT) in breast carcinoma cell lines ${ }^{30}$. Furthermore, EGF and BMP-4 cooperate to inhibit MMP-9 expression in cancer cells ${ }^{31}$. Additionally, EGFR signaling regulates the ability of MSCs to sustain cancer progression by triggering factors that promote neoangiogenesis and tumor cell migration ${ }^{32}$. Overall, metformin pretreatment of BM-MSCs provokes divergent results that may eventually promote osteogenesis and decreased metastasis via suppression of MMP9.

Several other factors were induced in response to metformin in HIP2 and KM76 BM-MSCs. ICAM-1/CD54 is a type of intercellular adhesion molecule present in leukocytes, endothelial cells and other cell types. BM-MSCs and Wharton Jelly-MSCs were reported to show high CD54 surface expression in contrast to low expression in ADSCs ${ }^{33}$. CD54 is upregulated specifically at the interphase between M1 macrophages and MSCs and this interaction increased the CD54-mediated immunosuppressive function of MSCs. Furthermore, the expression of RANTES was also found to be upregulated by CD54 ligation. IL-11 revealed a chemoattractive effect towards human BM-MSCs ${ }^{34}$. This interleukin showed an angiogenic effect by promoting the formation of longer tubule structures and higher amounts of junctions. Visfatin is a peptide that increases matrix mineralization and reduces collagen type I expression. Visfatin is involved in impaired bone remodeling at the adipose tissue/bone interface through induction of proinflammatory factors and dysregulated MMP/TIMP balance during MSC differentiation ${ }^{35}$. The role of visfatin for specific tumor types is variable and not fully characterized $^{36}$. The receptor for advanced glycation endproducts (RAGE) drives progression and metastasis of tumors through distinct mechanisms ${ }^{37}$. Advanced glycation endproducts (AGEs) inhibit the proliferation of MSCs, induce apoptosis and prevent differentiation into adipose tissue, cartilage, and bone ${ }^{38,39}$. AGEs were shown to increase proliferation, migration and invasion of the breast cancer cell line MDA-MB-23140. One important explanation of metformin's effect on diabetic complications could be its ability to reduce toxic dicarbonyls and $A G E s^{41}$. Thus, increased expression of RAGE is expected to have a minor role in presence of reduced amounts of AGEs.

Breast cancer cells stimulate the secretion of the chemokine CCL5 (also called: Regulated upon Activation, Normal T Cell Expressed and Presumably Secreted; RANTES) from MSCs, 
which then enhances their motility, invasion and metastasis. This enhanced metastatic ability is reversible and dependent on CCL5 signaling through the chemokine receptor CCR5 ${ }^{42}$. Dormant-like tumor-initiating cells do not respond to MSC signals, unlike actively dividing cancer cells which are supported by $\mathrm{MSCs}^{43}$. The secretome of MSC isolated from various tissues is variable but it includes mostly CCL2, CCL5, IL-6, TGF $\beta$, VEGF, which have been implicated in tumor growth and/or metastasis. CCL5 is overexpressed in response to metformin in HIP2 and KM76, in contrast to the low or unchanged expression of VEGF. In cocultures, metformin-stimulated ADSCs inhibited the expression of RUNX2, COL X, VEGF, MMP1, MMP3, and MMP13 in chondrocytes and increased the expression of tissue inhibitors of metalloproteinases (TIMP) TIMP1 and TIMP3 ${ }^{44}$. MSC have the potential to migrate through bone marrow endothelium and that this process involves MMP-2. Increased culture confluence impairs migration by upregulation of TIMP- $3^{45}$. Hypermethylation of TIMP3 in invasive breast cancer might be associated with high tumor grading and metastasis ${ }^{46}$. TIMP3 can form a stable complex with pro-MMP9, and has been shown to inhibit MMP9 activity ${ }^{47}$. Thus, increased expression of TIMP-3 of KM76 and W1951 in response to metformin seem to have an anti-invasive effect. VEGF165-transfected bone marrow MSCs promotes vascularization of tissue-engineered bone and ectopic osteogenesis but is here not responsive to metformin ${ }^{48}$. Again, metformin-modulated expression of CCL5/RANTES and TIMP3 provide contradictory results with possible prevention of metastasis by increased expression of this inhibitor of metalloproteinases.

Chemerin is a chemoattractant protein that acts as a ligand for the $G$ protein-coupled receptor CMKLR1. Increasing chemerin expression within the breast carcinoma TME can suppress growth by recruitment of NK and T cells ${ }^{49}$. Adipsin (also called complement factor $\mathrm{D} / \mathrm{CFD}$ ) is a serine protease synthesized by adipocytes capable of activating the alternative pathway of the complement, triggering the natural defense against infections. However, the growth of breast cancer xenotransplants was increased by co-transplantation with adipsinexpressing ADSCs in vivo ${ }^{50}$. Fibroblast growth factors (FGFs) enhance the proliferation of BMMSCs at high levels ${ }^{51}$. Treatment with FGF-2 rapidly induced activation of AKT and ERK. A fibrinogen matrix stimulates a two-fold increase in BM-MSC cell yield over plastic surfaces without affecting differentiation ${ }^{52}$. The significance of the multitude of adipokines altered in response to metformin is difficult to interpret but increase of osteogenic signals, activation of immune processes and inhibition of MMP9 is expected to be beneficial for patients ${ }^{53}$.

\section{Conclusion}

In conclusion, metformin-induced changes in the expression of adipokine-related proteins indicate partially contradictory effects on BM-MSCs and cancer cells. Overall, the metformin effects may lead to inhibition of tumor cell proliferation for specific cancer cell-BM-MSC pairs, significant inhibition of breast cancer cell migration and possibly increased osteogenic and anti-metastatic effects. The variability of the antitumor effects via BM-MSCs may be correlated to the reports describing reduced cancer incidence in patients receiving metformin and contradictory investigations failing to find a patient benefit of such treatment. 


\section{Acknowledgment}

We thank Dr. T. Hohenheim for continuing endorsement.

\section{Funding}

This research was partially funded by the "Medical Scientific Fund of the Mayor of the City of Vienna", grant number 19090 to Dr. C. Neumayer.

\section{Availability of data and materials}

Data and materials are available on reasonable request.

\section{Contributions}

G.H., C.L. and M.T. were responsible for the conceptualization; methodology was provided by A.P., B.R. and D.M.; software, A.P., B.R. and G.H. carried out the investigation; B.R. and A.P. were responsible for the validation, C.R. and C.S. provided the resources; C.N. acquired the funding; all author participated in drafting and correcting the manuscript. All authors have read and agreed to the submitted version of the manuscript.

\section{Ethics declarations}

\section{Ethics approval and informed consent to participate}

BM-MSCs were recovered from patients undergoing hip replacement with informed consent of the patients according to the Ethics Approval 366/2003 of the Ethics Committee of the Medical University of Vienna, Vienna, Austria. Informed consent has been obtained from all participating patients.

\section{Consent for publication}

Not applicable.

\section{Competing interests}

The authors declare that they have no competing interests. 


\section{References}

1. Harbeck, N. et al. Breast cancer. Nat. Rev. Dis. Primers 5 (1) ,66 (20019). doi: 10.1038/s41572-019-0111-2.

2. Marazzi, F. et al. 2020. Diagnosis and Treatment of Bone Metastases in Breast Cancer: Radiotherapy, Local Approach and Systemic Therapy in a Guide for Clinicians. Cancers (Basel) 12 (9), E2390. doi: 10.3390/cancers12092390.

3. Clements, ME. \& Johnson, R.W. Breast Cancer Dormancy in Bone. Curr. Osteoporos. Rep. 17 (5), 353-361 (2019). doi: 10.1007/s11914-019-00532-y.

4. Haider, M.T., Smit D.J. \& Taipaleenmäki, H. The Endosteal Niche in Breast Cancer Bone Metastasis. Front. Oncol. 10, 335 (2020). doi: 10.3389/fonc.2020.00335.

5. Casson, J., Davies, O.G., Smith, C.A., Dalby, M.J. \& Berry, C.C. Mesenchymal stem cellderived extracellular vesicles may promote breast cancer cell dormancy. J. Tissue Eng. 9, 2041731418810093 (2018). doi: 10.1177/2041731418810093.

6. Maumus, M., Rozier, P., Boulestreau, J., Jorgensen, C. \& Noël, D. Mesenchymal Stem CellDerived Extracellular Vesicles: Opportunities and Challenges for Clinical Translation. Front. Bioeng. Biotechnol. 8, 997 (2020). doi: 10.3389/fbioe.2020.00997.

7. Bianco, P. "Mesenchymal" stem cells. Annu. Rev. Cell Dev. Biol. 30, 677-704 (2014). doi: 10.1146/annurev-cellbio-100913-013132.

8. Saeedi, M. Biological Aspects and Clinical Applications of Mesenchymal Stem Cells: Key Features You Need to be Aware of. Curr. Pharm. Biotechnol. Online ahead of print (2020). doi: 10.2174/1389201021666200907121530.

9. Farahmand, L., Esmaeili, R., Eini, L. \& Majidzadeh-A K. The effect of mesenchymal stem cell-conditioned medium on proliferation and apoptosis of breast cancer cell line. J. Cancer Res. Ther. 14 (2), 341-344 (2018). doi: 10.4103/0973-1482.177213.

10. Roshan, M.H., Shing, Y.K. \& Pace, N.P. Metformin as an adjuvant in breast cancer treatment. SAGE Open Med. 7, 2050312119865114 (2019). doi: $10.1177 / 2050312119865114$.

11. Schulten, H.J. Pleiotropic Effects of Metformin on Cancer. Int. J. Mol. Sci. 19 (10), 2850 (2018). doi: 10.3390/ijms19102850.

12. Bailey, C.J. Metformin: Historical overview. Diabetologia 60 (9), 1566-1576 (2017). doi: 10.1007/s00125-017-4318-z.

13. De, A. \& Kuppusamy, G. Metformin in breast cancer: preclinical and clinical evidence. Curr. Probl. Cancer 44 (1), 100488 (2020). doi: 10.1016/j.currproblcancer.2019.06.003.

14. Teufelsbauer, M. et al. 2020. Effects of metformin on adipose-derived stromal cell (ADSC) - Breast cancer cell lines interaction. Life Sci. 261, 118371 (2020). doi: 10.1016/j.Ifs.2020.118371.

15. Hou, G. Clinical pathological characteristics and prognostic analysis of 1,013 breast cancer patients with diabetes. Breast Cancer Res. Treat 137 (3), 807-816 (2013). doi: 10.1007/s10549-012-2404-y. 
16. Chen, Y.C., Li, H. \& Wang, J. Mechanisms of metformin inhibiting cancer invasion and migration. Am. J. Transl. Res. 12 (9), 4885-4901 (2020). eCollection 2020.

17. Kang, Y.J., Jeong, H.C., Kim, T.E. \& Shin, K.H. Bioanalytical Method Using Ultra-HighPerformance Liquid Chromatography Coupled with High-Resolution Mass Spectrometry (UHPL-CHRMS) for the Detection of Metformin in Human Plasma. Molecules 25 (20), 4625 (2020). doi: 10.3390/molecules25204625.

18. Kinaan, M., Ding, H. \& Triggle, C.R. Metformin: An Old Drug for the Treatment of Diabetes but a New Drug for the Protection of the Endothelium. Med. Princ. Pract. 24 (5), 401-415 (2015). doi: 10.1159/000381643.

19. Mohamed-Ahmed, S. Adipose-derived and bone marrow mesenchymal stem cells: a donor-matched comparison. Stem Cell Res. Ther. 9 (1), 168 (2018). doi: 10.1186/s13287018-0914-1.

20. Mareschi, K. Multipotent mesenchymal stromal stem cell expansion by plating whole bone marrow at a low cellular density: a more advantageous method for clinical use. Stem Cells Int. 920581 (2012). doi: 10.1155/2012/920581.

21. Brown, M.J., Bahsoun, S., Morris, M.A. \& Akam, A.E.C. Determining Conditions for Successful Culture of Multi-Cellular 3D Tumour Spheroids to Investigate the Effect of Mesenchymal Stem Cells on Breast Cancer Cell Invasiveness. Bioengineering (Basel) 6 (4), 101 (2019). doi: 10.3390/bioengineering6040101.

22. Choi, J., Cha, Y.J. \& Koo, J.S., 2018. Adipocyte biology in breast cancer: From silent bystander to active facilitator. Prog. Lipid Res. 69, 11-20 (2018). doi: 10.1016/j.plipres.2017.11.002.

23. Wang, Y. 2017. Adiponectin regulates BMSC osteogenic differentiation and osteogenesis through the Wnt/ $\beta$-catenin pathway. Sci Rep. 7 (1), 3652 (2017). doi: 10.1038/s41598017-03899-z.

24. Lee, H.W. Adiponectin stimulates osteoblast differentiation through induction of COX2 in mesenchymal progenitor cells. Stem Cells 27 (9), 2254-62 (2009). doi: 10.1002/stem.144.

25. Min, S.Y. Diverse repertoire of human adipocyte subtypes develops from transcriptionally distinct mesenchymal progenitor cells. Proc. Natl. Acad. Sci. USA 116 (36),17970-17979 (2019). doi: 10.1073/pnas.1906512116.

26. Cui, E. Adiponectin inhibits migration and invasion by reversing epithelial-mesenchymal transition in non-small cell lung carcinoma. Oncol. Rep. 40 (3), 1330-1338 (2018). doi: 10.3892/or.2018.6523.

27. Han, H.H. Angiopoietin-2 promotes ER+ breast cancer cell survival in bone marrow niche. Endocr. Relat. Cancer 23 (8), 609-623 (2016). doi: 10.1530/ERC-16-0086.

28. Seib, F.P., Franke, M., Jing, D., Werner, C. \& Bornhäuser, M. Endogenous bone morphogenetic proteins in human bone marrow-derived multipotent mesenchymal stromal cells. Eur. J. Cell. Biol. 88 (5), 257-271 (2009). doi: 10.1016/j.ejcb.2009.01.003.

29. Sekiya, I., Larson, B.L., Vuoristo, J.T., Reger, R.L. \& Prockop, D.J. Comparison of effect of BMP-2, -4 , and -6 on in vitro cartilage formation of human adult stem cells from bone 
marrow stroma. Cell Tissue Res. 320 (2), 269-276 (2005). doi: 10.1007/s00441-004-10753.

30. Choi, S. BMP-4 enhances epithelial mesenchymal transition and cancer stem cell properties of breast cancer cells via Notch signaling. Sci. Rep. 9 (1), 11724 (2019). doi: 10.1038/s41598-019-48190-5.

31. Laulan, N.B. \& St-Pierre, Y. Bone morphogenetic protein 4 (BMP-4) and epidermal growth factor (EGF) inhibit metalloproteinase-9 (MMP-9) expression in cancer cells. Oncoscience. 2 (3), 309-316 (2015). doi: 10.18632/oncoscience.144.

32. De Luca, A. Role of the EGFR ligand/receptor system in the secretion of angiogenic factors in mesenchymal stem cells. J. Cell Physiol. 226 (8), 2131-2138 (2011). doi: 10.1002/jcp.22548.

33. Amable, P.R., Teixeira, M.V., Carias, R.B., Granjeiro, J.M. \& Borojevic, R. Protein synthesis and secretion in human mesenchymal cells derived from bone marrow, adipose tissue and Wharton's jelly. Stem Cell Res. Ther. 5 (2), 53 (2014). doi: 10.1186/scrt442.

34. Gabrielyan, A., Quade, M., Gelinsky, M. \& Rösen-Wolff, A. IL-11 and soluble VCAM-1 are important components of Hypoxia Conditioned Media and crucial for Mesenchymal Stromal Cells attraction. Stem Cell Res. 45, 101814 (2020). doi: 10.1016/j.scr.2020.101814.

35. Tsiklauri, L. Visfatin alters the cytokine and matrix-degrading enzyme profile during osteogenic and adipogenic MSC differentiation. Osteoarthritis Cartilage 26 (9), 1225-1235 (2018). doi: 10.1016/j.joca.2018.06.001.

36. Lin, T.C. The role of visfatin in cancer proliferation, angiogenesis, metastasis, drug resistance and clinical prognosis. Cancer Manag. Res. 11, $3481-3491$ (2019). doi: 10.2147/CMAR.S199597.

37. Kwak, T. Targeting of RAGE-ligand signaling impairs breast cancer cell invasion and metastasis. Oncogene 36, 1559-1572 (2017). doi: 10.1038/onc.2016.324.

38. Kume, S. Advanced glycation end-products attenuate human mesenchymal stem cells and prevent cognate differentiation into adipose tissue, cartilage, and bone. J. Bone Miner Res. 20 (9), 1647-1658 (2005). doi: 10.1359/JBMR.050514.

39. Lu, Y.Q., Lu, Y., Li, H.J. \& Chen,g X.B. Effect of advanced glycosylation end products (AGEs) on proliferation of human bone marrow mesenchymal stem cells (MSCs) in vitro. In Vitro Cell Dev. Biol. Anim. 48 (9), 599-602 (2012). doi: 10.1007/s11626-012-9551-7.

40. Sharaf, $H$. Advanced glycation endproducts increase proliferation, migration and invasion of the breast cancer cell line MDA-MB-231. Biochim. Biophys. Acta 1852 (3), 429-441 (2015). doi: 10.1016/j.bbadis.2014.12.009.

41. Beisswenger, P. \& Ruggiero-Lopez, D. Metformin inhibition of glycation processes. Diabetes Metab. 29 (4 Pt 2), 6S95-103 (2003). doi: 10.1016/s1262-3636(03)72793-1.

42. Karnoub, A.E. Mesenchymal stem cells within tumour stroma promote breast cancer metastasis. Nature 449 (7162), 557-63 (2007). doi: 10.1038/nature06188. 
43. Zimmerlin, L., Park, T.S., Zambidis, E.T., Donnenberg, V.S. \& Donnenberg, A.D. Mesenchymal stem cell secretome and regenerative therapy after cancer. Biochimie 95 (12), 2235-2245 (2013). doi: 10.1016/j.biochi.2013.05.010.

44. Park, M.J. Metformin Augments Anti-Inflammatory and Chondroprotective Properties of Mesenchymal Stem Cells in Experimental Osteoarthritis. J. Immunol. 203 (1), 127-136 (2019). doi: 10.4049/jimmunol.1800006.

45. De Becker, A. Migration of culture-expanded human mesenchymal stem cells through bone marrow endothelium is regulated by matrix metalloproteinase- 2 and tissue inhibitor of metalloproteinase-3. Haematologica 92 (4), 440-449 (2007). doi: 10.3324/haematol.10475.

46. Lui, E.L., Loo, W.T., Zhu, L., Cheung, M.N. \& Chow, L.W. DNA hypermethylation of TIMP3 gene in invasive breast ductal carcinoma. Biomed Pharmacother. 59 (Suppl 2), S363-365 (2005). doi: 10.1016/s0753-3322(05)80079-4

47. Nagase, H., Visse, R. \& Murphy, G. Structure and function of matrix metalloproteinases and TIMPs. Cardiovasc. Res. 69 (3), 562-573 (2006). doi: 10.1016/j.cardiores.2005.12.002.

48. Liu, B., Li, X., Liang, G. \& Liu, X. VEGF expression in mesenchymal stem cells promotes bone formation of tissue-engineered bones. Mol. Med. Rep. 4 (6), 1121-1126 (2011). doi: 10.3892/mmr.2011.559.

49. Pachynski, R.K. Chemerin Suppresses Breast Cancer Growth by Recruiting Immune Effector Cells into the Tumor Microenvironment. Front. Immunol. 10, 983 (2019). doi: 10.3389/fimmu.2019.00983.

50. Goto, H.2019. Adipose-derived stem cells enhance human breast cancer growth and cancer stem cell-like properties through adipsin. Oncogene 38 (6), 767-779. doi: 10.1038/s41388-018-0477-8.

51. Eom, Y.W. The role of growth factors in maintenance of stemness in bone marrow-derived mesenchymal stem cells. Biochem. Biophys. Res. Commun. 445 (1), 16-22 (2014). doi: 10.1016/j.bbrc.2014.01.084.

52. Kisiday, J.D. Expansion of mesenchymal stem cells on fibrinogen-rich protein surfaces derived from blood plasma. J. Tissue Eng. Regen. Med. 5 (8), 600-611 (2011). doi: 10.1002/term.352.

53. Teufelsbauer, M. Interaction of Adipose-Derived Stromal Cells with Breast Cancer Cell Lines. Plast. Reconstr. Surg. 144 (2), 207e-217e (2019). doi: 10.1097/PRS.0000000000005839. 